\title{
Plasma-density evolution in compact polyacetal capillary discharges
}

\author{
F. G. Tomasel, J. J. Rocca, O. D. Cortázar, and B. T. Szapiro \\ Electrical Engineering Department, Colorado State University, Fort Collins, Colorado 80523
}

R. W. Lee

Lawrence Livermore National Laboratory, Livermore, California 94550

(Received 15 December 1992)

\begin{abstract}
We have measured the temporal evolution of the electron density of plasmas produced in polyacetal capillaries with diameters between 0.5 and $1.5 \mathrm{~mm}$ excited by 110-ns full-width-at-half-maximum discharge pulses with currents between 13 and $42 \mathrm{kA}$. The electron density was determined from Starkbroadened line profiles of the $4 f-3 d \mathrm{O}$ vi transition taking into account opacity effects. The electron density was found to increase continuously during the rise of the current pulse, and to decrease near the end of the current pulse, when a drop in plasma temperature causes the degree of ionization of the plasma to decrease. The peak plasma density in a $1-\mathrm{mm}$ capillary excited by a $24-\mathrm{kA}$ pulse was measured to be $5 \times 10^{19} \mathrm{~cm}^{-3}$. The plasma density was observed to increase linearly with discharge energy from $7.5 \times 10^{18} \mathrm{~cm}^{-3}$ for a $5-\mathrm{J}$ discharge to $5 \times 10^{19} \mathrm{~cm}^{-3}$ for a $30-\mathrm{J}$ discharge in a $1.5-\mathrm{mm}$-diam. capillary.

PACS number(s): 52.80. $-\mathrm{s}, 42.55 . \mathrm{Vc}$
\end{abstract}

\section{INTRODUCTION}

In this paper, we report measurements of the temporal evolution of the electron density in polyacetal $\left(\mathrm{CH}_{2} \mathrm{O}\right)_{n}$ capillary discharges. Capillary-discharge plasmas are intense sources of soft $\mathrm{x}$-ray radiation that have been used in spectroscopy, contact microscopy, and lithography $[1-3]$. They have also been investigated as sources of continuum radiation at longer wavelengths [2]. Previous studies of capillary discharges have included timeintegrated measurements of the plasma parameters. Bogen et al. [2] suggested, based on measurements of the continuum intensity at two different wavelengths, that the electron density in polyethylene capillaries 0.5 to 2 $\mathrm{mm}$ diameter excited by 60-ns full-width-at-halfmaximum (FWHM) discharge pulses from a 50-nF capacitor charged to $35 \mathrm{kV}$ increases continuously as a function of time during the first half cycle of the current pulse. From these experiments, values of the electron density of $2.5 \times 10^{19} \mathrm{~cm}^{-3}$ from continuum-intensity measurements and $1.5 \times 10^{19} \mathrm{~cm}^{-3}$ from Stark broadening of $\mathrm{C}_{\mathrm{v}}$ lines were derived, but no detailed measurements of the temporal evolution of the electron density were reported.

More recently, the need for more compact and higherefficiency soft-x-ray lasers has brought renewed attention to capillary-discharge plasmas [4-11]. The possibility of developing soft-x-ray recombination lasers based on fastdischarge excitation of capillary plasma columns has been proposed [4]. In this scheme, a high-current pulse creates a highly ionized plasma column that rapidly cools and recombines at the end of the current pulse by heat losses produced by radiation and electron heat conduction to the capillary walls. This sudden recombination could possibly produce population inversions and amplification. In recombination laser schemes, the electron density at the time of maximum recombination is a very important parameter, as the collisional recombina- tion rate and the gain increase sharply as a function of the plasma density. However, there exists an upper density limit beyond which the population inversion is destroyed by the electron collisional mixing of the laser upper- and lower-level populations. This explains the importance of time-resolved measurements of the electron density.

Of particular interest are capillary plasmas containing carbon for the generation of population inversions in the $m=2$ to $m=3$ transition of hydrogenic carbon, in which gain at $18.2 \mathrm{~nm}$ has been obtained in laser-created plasmas [12]. Recently, short bursts of radiation at $18.2 \mathrm{~nm}$ have been reported to be observed in $0.5-\mathrm{mm}$ - and 1mm-diam polyacetal capillaries excited by current pulses from a $100-\mathrm{nF}$, low-voltage $(7-16 \mathrm{kV})$, low-inductance discharge [6]. These bursts, which were detected utilizing a scintillator-photomultiplier combination, were observed to increase in intensity with capillary length, and consequently have been interpreted as amplified spontaneous emission of the $m=2$ to $m=3$ transition of C VI. However, the reported timing at which the bursts appeared, the vicinity of the peak of the second half cycle of the current pulse, does not correspond to the time at which maximum recombination is expected to occur. Again, additional diagnostics and measurements of the evolution of the plasma parameters become an important aid to the interpretation of the results of these experiments.

Here we report results of time-resolved extremeultraviolet (XUV) spectroscopy of a polyacetal capillary discharge having similar parameters to those of the experiment described above. Our measurements were conducted in capillaries $0.5,1$, and $1.5 \mathrm{~mm}$ diameter at excitation voltages between 10 and $25 \mathrm{kV}$. The temporal evolution of the electron density was determined by comparing linewidth measurements $\mathrm{O}$ VI $52-\mathrm{nm} 4 f-3 d$ transition to those corresponding to the calculated line profiles discussed in Sec. V [13]. The spectra line broadening of this 
transition in lithiumlike ions has been previously studied by several authors [14-16].

\section{GENERATION OF THE CAPILLARY PLASMA}

The discharge setup is the same described in Ref. [11]. The capillaries are excited by discharging a lowinductance capacitor array. This array is composed of 32 ceramic capacitors connected in parallel, forming a rectangle $53 \mathrm{~cm}$ high by $66 \mathrm{~cm}$ wide, with the capillary being in the center. The total capacitance is $100 \mathrm{nF}$ and the total discharge circuit inductance is $23 \mathrm{nH}$ when $1-\mathrm{mm}-$ diam, 1-cm-long capillaries are used. The capacitors are connected to the capillary electrodes through a parallelplate transmission line, and the total assemblage is immersed into an acrylic box containing transformer oil to avoid external breakdown at the higher voltages. The discharge electrodes are made out of graphite, and are bored axially to a diameter of $3 \mathrm{~mm}$ to allow for the exit of the radiation from the capillary plasma.

The capillary was evacuated to a pressure below $2 \times 10^{-5}$ Torr utilizing a turbomolecular pump. Following evacuation, the capillary serves as an open high- voltage vacuum switch, and the capacitor bank is charged to the desired voltage utilizing a dc power supply. To trigger the capillary discharge, a $100-\mathrm{kV}$ negative pulse from a small $(0.2 \mathrm{nF})$ Marx capacitor bank is applied to a third electrode placed in the proximity of the grounded cathode electrode. In the experiment reported herein, the discharge was operated in the voltage range from $10 \mathrm{kV}$ to $25 \mathrm{kV}$, corresponding to peak currents between 13 nd $42 \mathrm{kA}$ through a 1 -mm-diam capillary. The discharge current was monitored using a Rogowsky coil. The 110-ns FWHM discharge current pulse corresponding to a $15-\mathrm{keV}$ discharge is included in Fig. 1.

\section{TEMPORAL EVOLUTION OF THE XUV EMISSION}

The end-on emission from the discharge was collected and focused by a cylindrical aluminum mirror into the slit of a 1-m focal length, grazing-incidence vacuum spectrograph provided with a $600-1$ ines $/ \mathrm{mm}$ gold-coated grating and a windowless multichannel plate (MCP) intensified diode array detector. The radiation intensity was sufficient to obtain single-shot spectra with an entrance slit of $30 \mu \mathrm{m}$ and a temporal resolution of approxi-
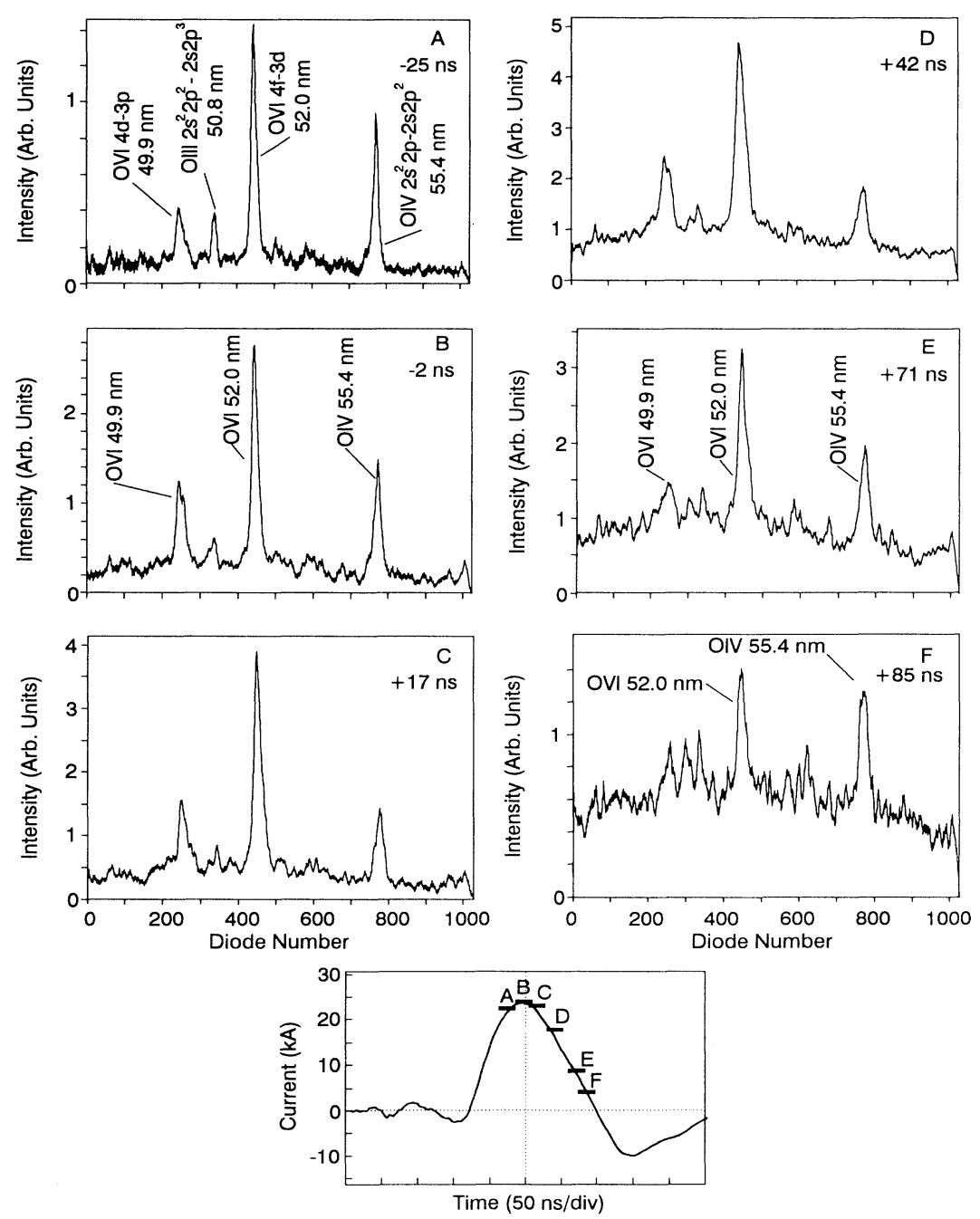

FIG. 1. Sequence of time-resolved spectra corresponding to a 1-mm-diam, 1-cm-long polyacetal capillary excited by the current pulse depicted on the bottom center. The spectra cover the range between 47 and $57 \mathrm{~nm}$. 
mately $5 \mathrm{~ns}$. Temporal evolution was obtained by gating the gain of the MCP intensified with a short high-voltage pulse. To determine the timing of the gate pulse with respect to the current pulse, both pulses were acquired using a dual-channel $10^{9}$-sample-per-second digitizing oscilloscope.

Figure 1 shows the time evolution of the axial soft-xray emission from a $15-\mathrm{kV}$ discharge in a 1-mm-diam, 1$\mathrm{cm}$-long capillary in the spectral region from 47 to 57 $\mathrm{nm}$. The timing of each spectrum with respect to the peak of the current pulse is specified in the figure. The earliest time at which a spectrum could be obtained was limited by the intrinsic delay of the gating circuit. From this sequence of spectra, it can be seen that the most intense line in this spectral region is the OVI $4 f-3 d$ transition at $52 \mathrm{~nm}$ which, being sensitive to Stark broadening, was selected to determine the electron density. The O VI $4 d-3 p$ transition at $49.9 \mathrm{~nm}$ is observed to the left of the $4 f-3 d$ line. As described in Sec. VI, the peak-intensity ratio of these two transitions was utilized to determine the optical depth of the $4 f-3 d$ line, for the purpose of evaluating the contribution of opacity to the broadening of this line. The $\mathrm{O}$ IV $2 s^{2} 2 p^{2} P^{0}-2 s 2 p^{22} P(\lambda=55.4 \mathrm{~nm})$ transition is also observed at the right of the spectrum. The ratio of the intensity of the OVI and O IV lines, which is indicative of the degree of ionization of the plasma, is observed to be maximum $42 \mathrm{~ns}$ after the peak of the current. After this time, the intensity of the OVI transition decreases significantly and, at the end of the current pulse, the $O$ IV transition becomes comparable in intensity to the $\mathrm{O}$ VI lines. Lines from ions with lower degrees of ionization, like $\mathrm{O}$ III, are also seen at the beginning of the current pulse. The simultaneous appearance of lines from ions with very different ionization degrees suggests the existence of a hotter plasma on the axis surrounded by a cooler plasma close to the capillary walls. The electron-density measurements described herein are spatially integrated. However, since the electron-density values were obtained analyzing the line profile of the $\mathrm{O}$ VI $4 f-3 d$ transition, they are representative of the central region of the plasma column, where the OVI ions are present.

The predominance of the OVI lines near the peak of the first half cycle of the current is also observed in other regions of the spectrum. Figure 2 shows a sequence of time-resolved spectra in the wavelength range between 10
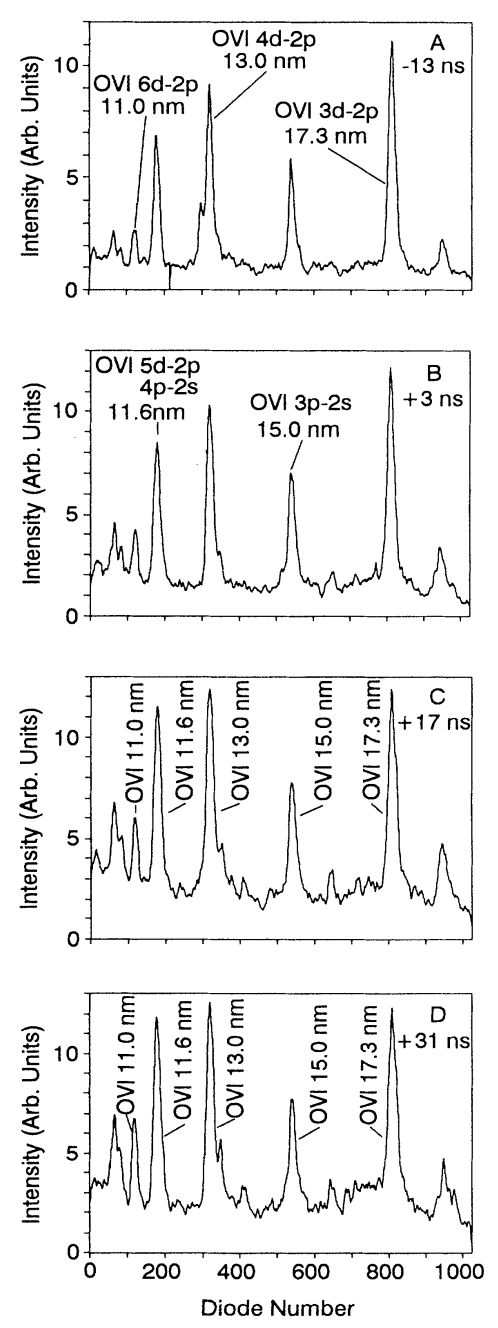
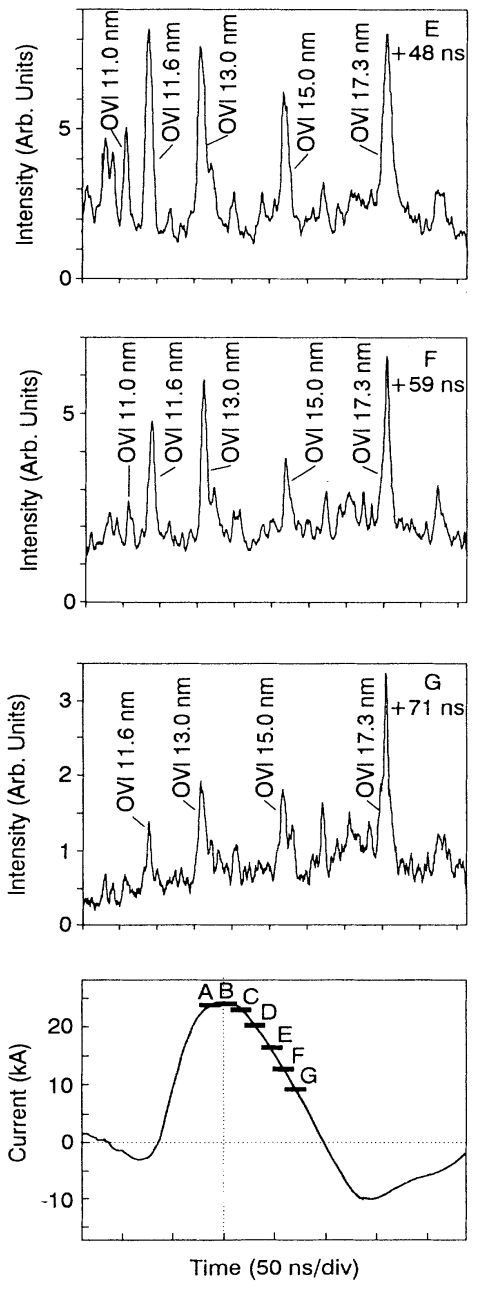

FIG. 2. Sequence of time-resolved $x$-ray spectra from $15-\mathrm{kV}$ discharges in 1-mm-diam, $1-\mathrm{cm}$-long polyacetal capillaries covering the region between 10 and $18 \mathrm{~nm}$. The timing of each spectrum relative to the current pulse is indicated. 


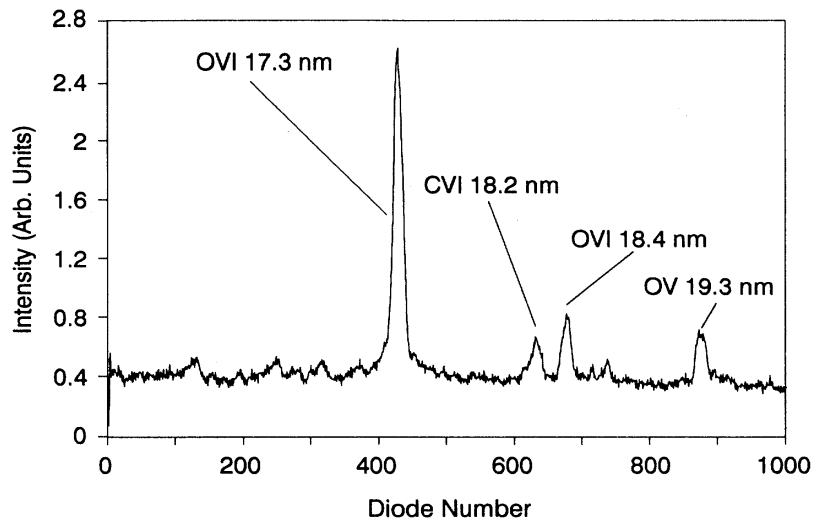

FIG. 3. Time-resolved spectra from 15.4 to $20 \mathrm{~nm}$ corresponding to the time of the peak of the current pulse in a $16-\mathrm{kV}$ discharge through a 1-mm-diam, 1-cm-long polyacetal capillary.

and $18 \mathrm{~nm}$. In several of these spectra, the intensity of the $4 d-2 p$ and $5 d-2 p$ transitions appears to be anomalously intense with respect to the $3 d-2 p$ line. This phenomenon is not associated to population inversions, but is rather caused by reabsorption of the O VI $3 d-2 p$ 17.3-nm lines. The electron-density measurements discussed herein indicate that the plasma density greatly exceeds the values at which inversions between these $\mathrm{O}$ VI levels are possible. In these spectra, the strong $\mathrm{O}$ VII $3 d$ $2 p$ transition at $12.85 \mathrm{~nm}$ is not observed. This is in marked contrast with the polyacetal capillary spectra we have obtained from a faster (11-ns risetime, 25-ns FWHM) high-power discharge, in which this O viI transition has comparable or even greater intensity than the O VI $4 d-2 p$ line at $13 \mathrm{~nm}$ [10]. On the slower polyacetal discharges discussed herein, carbon lines are observed to be much weaker than oxygen lines. The C VI Balmer $\alpha$ line is only observed at discharge voltages above $10 \mathrm{kV}$ in the $0.5-\mathrm{mm}$-diam capillaries and at voltages above $15 \mathrm{kV}$ in the 1-mm-diam capillaries. Figure 3 shows that the emission from the CVI 18.2-nm transition for a $16-\mathrm{kV}$ discharge through a 1-mm-diam capillary, which was observed near the peak of the first half cycle of the current pulse, is nevertheless weak in comparison with the $\mathrm{O}$ VI 17.3-nm line. This is in contrast to discharges in polyethylene capillaries, in which strong emission from C VI is observed [11]. In the plasmas discussed herein, the C VI lines completely vanish by the end of the first halfcycle of the current pulse. During the second half cycle of the current, the reduced excitation and the increased mass accumulated from the continuous ablation of the capillary walls result in a cooler plasma with insignificant radiation from highly ionized species.

\section{LINE-PROFILE MEASUREMENTS}

In order to determine the electron density and to study its temporal evolution, we measured the line profile of the $\mathrm{O}$ VI $4 f-3 d$ transition at different times during the current pulse. The spectra were acquired activating the $\mathrm{MCP}$ intensifier with a fast pulse to obtain a temporal resolution of approximately $5 \mathrm{~ns}$. The observed line profiles are a result of the contributions from Stark broadening, Doppler broadening, and instrumental broadening, as well as optical-depth effects. To determine the contribution from the instrument, the line profile of the $\mathrm{He}$ I $1 s^{2} S-1 s 2 p{ }^{1} P^{0}$ transition at $58.4 \mathrm{~nm}$, from a $2-\mathrm{mA}$, low-pressure dc capillary-discharge plasma having dimensions similar to those of the polyacetal discharges, was measured. In this low-density, low-temperature plasma, the Stark and Doppler broadening for this transition are negligibly small compared to instrumental broadening, allowing for a measurement of the instrumental line profile. The instrumental linewidth at that wavelength was determined to be $1.15 \AA$.

A measured line profile of the 52-nm O VI emission corresponding to a $15-\mathrm{kV}$ discharge through a 1-mm-diam, 1-cm-long polyacetal capillary is shown in Fig. 4. The spectrum was obtained $70 \mathrm{~ns}$ after the maximum of the current pulse. The line profile is asymmetric and cannot be fitted with a single Voigt profile. The shoulder on the long-wavelength side is caused by the emission from the $4 d-3 d$ O vi dipole-forbidden transition. At high plasma densities, the ion-produced microfield mixes the energy levels, resulting in the appearance of the $4 d-3 d$ dipoleforbidden component close to the strong $4 f-3 d$ dipoleallowed line. This $\Delta l=0$ transition has been observed in lithiumlike carbon (C IV) and in lithiumlike nitrogen $(\mathrm{N} v)$ in a gas liner pinch [14]. Emission from the C VI $m=4$ to $m=3$ transition at $52.1 \mathrm{~nm}$, which could also contribute to the long-wavelength side of the line profile, is likely to be small, since, as mentioned before, all C VI transitions are observed to be very weak in these lowvoltage polyacetal capillary discharges. The measured line shapes are in good agreement with the calculated line profiles described in Sec. V.

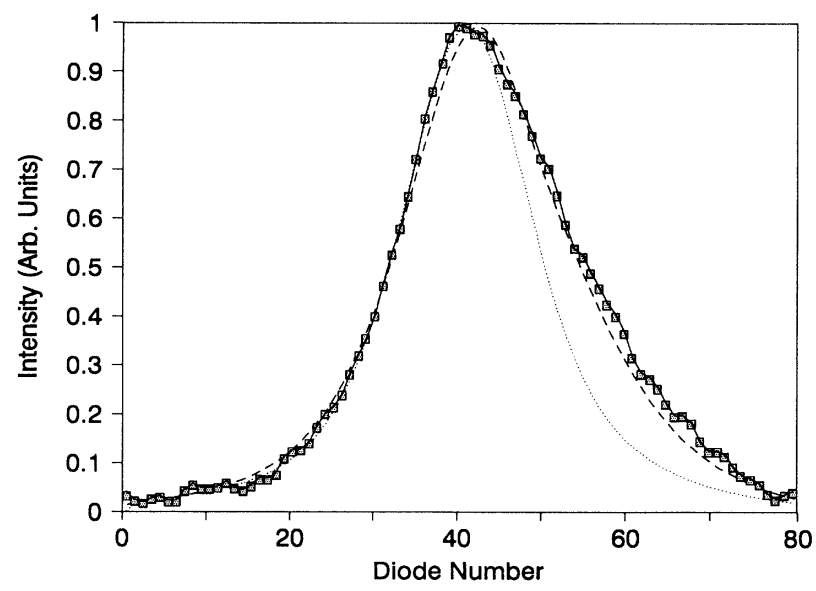

FIG. 4. Measured line profile of the $4 f-3 d$ O VI transition (solid line) corresponding to $70 \mathrm{~ns}$ after the maximum of the current pulse in a $15-\mathrm{kV}$ discharge through a 1-mm-diam capillary. The dashed line is the calculated line profile corresponding to $N_{e}=2 \times 10^{19} \mathrm{~cm}^{-3}, T_{e}=30 \mathrm{eV}, Z_{p}=3$ convolved with the instrumental line shape. The dotted line is a fitting of the measured line profile using a single Voigt profile. 


\section{CALCULATION OF THE O vi $4 f$-3 $d$ LINE PROFILES}

The model used to calculate the profile of spectral lines emitted by multielectron emitters in the capillary plasma is now described. In rough outline, the Stark broadening is included in the model by using the static-ion approximation and an impact approximation for the electrons [17]. The atomic data required for the line-shape calculation are extracted from an atomic-structure code [18] and prepared as the data necessary for the excited and lower level of the radiative transition.

Three main mechanisms affects the broadening of the emission lines of complex ions. The Doppler broadening produced by thermal motion of the radiating ions is generally the dominant mechanism in low-density plasmas for emitters having a moderate core charge $Z$. For increasing $Z$ values, spontaneous emission is enhanced, roughly as $Z^{4}$, and natural broadening may well exceed Doppler broadening. For higher plasma densities, the plasma fields generally broaden the line by the Stark effect, and Stark broadening may then govern the line shape. The latter is the case in the capillary-discharge plasma studied here. In the line-shape calculations presented here, all three effects are included. The line profile of the multielectron Li-like oxygen emitter is calculated for a selected spectral range, and tests were performed to ensure that sensitivity of the line shape to vari- ations in both the mean plasma charge and temperature were small.

The model used for the description of the Stark effect assumes that the quasistatic ion microfield approximation and the model of a binary-collision electronic operator are valid for this plasma and radiator combination. In our static-ion model, an average is performed over a static microfield distribution obtained by the adustableparameter-exponential-approximation (APEX) method [19], which allows rapid and accurate calculations. On the one hand, an electronic collision operator can safely be used to describe the regions near the line center. On the other hand, neglecting ion motion requires that the width of the line be much larger than the typical fluctuation frequency of the ion microfield. Since the breakdown of the static approximation has been found to occur for hydrogenic emitters in dense plasma conditions $[20,21]$, careful check for the validity of this approximation has been performed for the transitions calculated.

The calculated line shapes have been compared to the experimental works of Ref. [22], where the Li-like line shapes of elements from $Z=6$ to $Z=10$ have been measured, along with independent determination of the electron density and temperature. Our calculations compared well to the experimental FWHM for electron densities $\geq 10^{18} \mathrm{~cm}^{-3}$ within the experimentally quoted error of $20 \%$. Since in the present work higher densities are determined, these results lend confidence to the use of the calculated line shapes as a diagnostic tool.
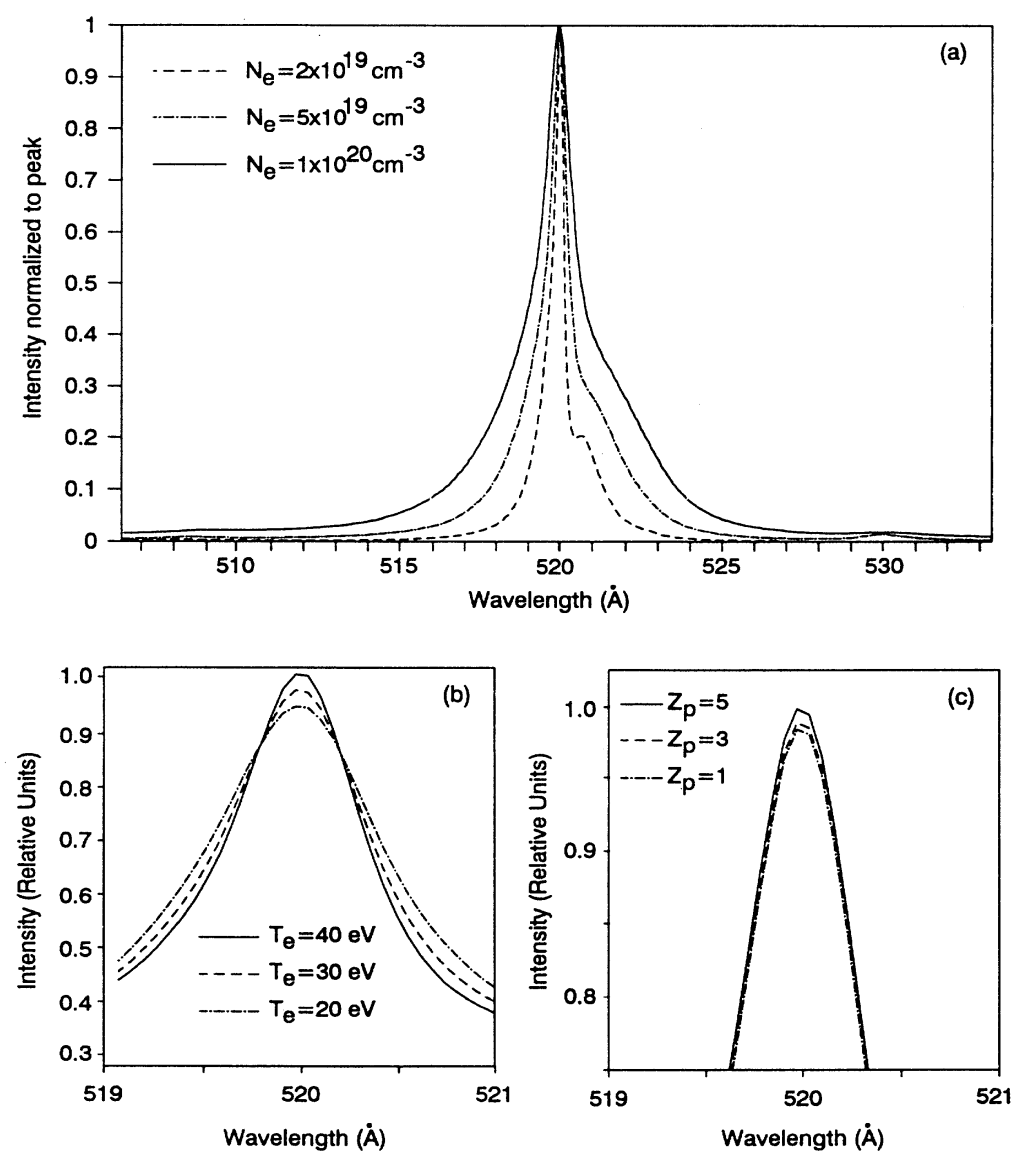

FIG. 5. Analysis of the sensitivity of the line profile to changes in the plasma parameters. (a) Calculated line profiles for $T_{e}=30 \mathrm{eV}$ and $Z_{p}=3$, corresponding to three different electron densities. (b) Line profiles for $N_{e}=1 \times 10^{20} \mathrm{~cm}^{-3}, Z_{p}=3$ at different temperatures. (c) Computed profiles for $N_{e}=1 \times 10^{20}$ $\mathrm{cm}^{-3}, \quad T_{e}=30 \mathrm{eV}$, and three different perturbing-ion charges. The line profile is observed to be quite sensitive to changes in the electron density, while changes in the profiles for cases (b) and (c) are only noticeable near the peak. 
The calculated optically thin line profiles for electron densities of $2 \times 10^{19} \mathrm{~cm}^{-3}, 5 \times 10^{19} \mathrm{~cm}^{-3}$, and $1 \times 10^{20}$ $\mathrm{cm}^{-3}$ are shown in Fig. 5(a). All three Stark-broadened line profiles contain Doppler convolutions for a temperature of $30 \mathrm{eV}$ (assumed based on the dominance of $\mathrm{O} \mathrm{VI}$ lines), and were computed considering that the charge of the perturbing ions is $Z_{p}=3$. In agreement with the measured profiles, the calculated line shapes show a shoulder in the long-wavelength side, corresponding to the contribution of the $4 d-3 d$ forbidden transition. Figures $5(\mathrm{~b})$ and 5(c) summarize the results of a sensitivity study of the line profile on variations of the temperature and effective charge of the perturbing ion. These figures, $5(\mathrm{~b})$ and $5(\mathrm{c})$, show that the line profiles are sensitive to changes in the electron density and are quite insensitive to changes in the other parameters. To determine the electron density, the optically thin calculated line profiles were first corrected for opacity effects, as described below. The resulting optically thick line profiles were then convolved with the instrumental line profile and the result was compared to the measured line shapes.

\section{OPTICAL-DEPTH CALCULATIONS}

At high densities, reabsorption of the radiation can be a significant source of additional broadening for the $4 f$ $3 d$ line, and consequently the effects of opacity need to be considered in determining the electron density from the measured line profiles.

Knowledge to the optical depth $\tau$ allows for the computation of the opacity-broadened line profile $I(\lambda)$ from the optically thin line profile $\phi(\lambda)$ :

$$
I(\lambda)=I_{0}\left\{1-\exp \left[-\tau^{\prime} \phi(\lambda)\right]\right\},
$$

with $\tau^{\prime}=\tau / \phi(\lambda)=\left(\pi e^{2} / m c\right) N_{L} l f_{L U}$, and where the constants have the usual meaning, $L$ and $U$ indicate the upper and lower level of the transition, $N_{L}$ is the population of the lower level of the transition, $l$ is the optical path length, and $f_{L U}$ is the absorption oscillator strength.

An unconstrained procedure of fitting the calculated line profiles to the measured line profiles with both the electron density and the optical depth as completely free parameters can yield large uncertainties. Instead, our approach consisted in independently determining the temporal evolution of the optical depth, or more precisely the $N_{L} l$ product, from the measured peak-intensity ratio of the $4 f-3 d$ and $4 d-3 p(\lambda=49.9 \mathrm{~nm}) \mathrm{O}$ VI lines. For this purpose, the ratio of the peak intensity of these lines was computed as a function of the optical depth of the $4 f-3 d$ line, with the electron density as parameter. Spectra synthesized by the model described in Sec. V were used to determine these peak-intensity ratios. For each value of the optical depth, synthesized spectra corresponding to various electron densities were broadened according to Eq. (1), utilizing the relative value of the populations of the $3 d$ and $3 p$ levels calculated using a kinetics model for oxygen, including detail on the lithium-, helium-, and hydrogenlike ions [23]. It was found that for the entire electron-density range of interest, the ratio between the populations of the $3 d$ and $3 p$ levels is calculated to be within $15 \%$ of the value corresponding to the ratio of the statistical weights, due to the large collisional mixing of these levels. As shown in Fig. 6, the peak-intensity ratio is rather insensitive to variations of the electron density in the range of interest, but depends strongly on the optical depth. The density dependence of the peak-intensity ratio is caused by the combination of the dipoleforbidden components which, sharing the oscillator strength with the allowed transitions, grow in intensity as the density increases. The close proximity of the two lines allows the assumption of a flat response of the instrument over this relatively small wavelength range. We verified that the spatial response of the MCP detector was also practically flat between the channels that coincide with each of the two lines.

The temporal evolution of the peak-intensity ratio for the above-mentioned lines, shown in Fig. 1 for the case of a $15-\mathrm{kV}$ discharge through a 1-mm-diam capillary, was observed to be similar in all the discharges studied. Figure 1 shows that this line ratio, which corresponds to a nearly optically thin plasma at the beginning of the current pulse, decreases with time, reaching a minimum $42 \mathrm{~ns}$ after the peak, and increases thereafter. The minimum $4 f-3 d$ to $4 d-3 p$ intensity ratio was measured to be about 2, which corresponds to an optical depth for the $4 f-3 d$ line of $3-4$ at line center. Line ratios as small as 1.6 , corresponding to an optical depth of 6-6.5, were measured for the $0.5-\mathrm{mm}$ capillaries. Note that for a given peak-intensity ratio in the range where the experimental ratios were measured, Fig. 6 shows that a variation of nearly an order of magnitude in the value of the electron density produces only a change of the order of unity in the value of the optical depth at line center, thereby allowing for the determination of the optical depth within an acceptable error range.

In fitting the measured line profiles with the opacitycorrected calculated line profiles, the optical depth was allowed to vary within the range determined by the corre-

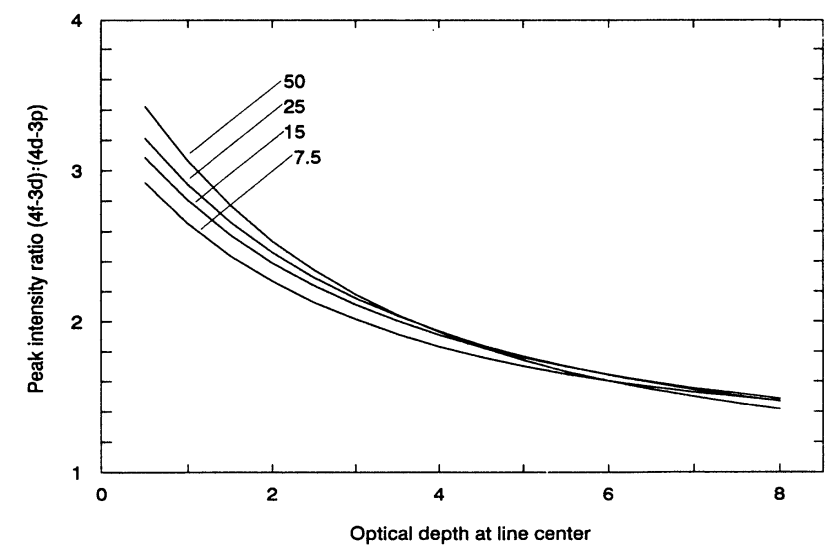

FIG. 6. Calculated peak-intensity ratios for the O VI $4 f-3 d$ to $4 d-3 p$ lines as a function of optical depth of the $4 f-3 d$ line at line center, with the electron density as parameter. The electron density is indicated in units of $1 \times 10^{18} \mathrm{~cm}^{-3}$. To allow direct comparison with the peak intensity of the experimental lines, the calculated line profiles were convolved with the instrumental profile prior to calculating the peak-intensity ratio. 
sponding peak-intensity ratio of Fig. 6, and the electron density was left as a free parameter. The shape of the computed line profiles convolved with the instrumental line function and corrected by absorption is in good agreement with the measured line profiles, as shown in Fig. 4 for an electron density of $2 \times 10^{19} \mathrm{~cm}^{-3}$.

\section{ELECTRON-DENSITY EVOLUTION}

The electron density was observed to follow a similar evolution in discharges conducted in all capillary diameters investigated: $0.5,1$, and $1.5 \mathrm{~mm}$. The electron density was measured to increase during the rise of the current pulse, to reach a maximum after the peak of the current, and to decrease towards the end of the first half cycle of the pulse. The maximum density was observed to occur later in time in the larger-diameter capillaries. While in the $0.5-\mathrm{mm}$ capillaries the maximum density occurs approximately $35 \mathrm{~ns}$ after the peak of the current, in the 1mm capillaries it occurs between 50 and $70 \mathrm{~ns}$ after the peak current is reached.

The measured electron-density evolution in a $1-\mathrm{mm}$ diam, 1-cm-long capillary excited by a $15-\mathrm{kV}$ pulse is illustrated in Fig. 7. At 30 ns before the peak of the current pulse, the earliest time at which spectra could be obtained, the density is measured to be $1 \times 10^{19} \mathrm{~cm}^{-3}$. The plasma density is observed to increase as a function of time, reaching a maximum of approximately $5 \times 10^{19}$ $\mathrm{cm}^{-3}$ occurring about 50-70 ns after the peak of the current pulse. From comparison with Fig. 1, it can be observed that the increase in the electron density follows the increase of the degree of ionization of the plasma. Towards the end of the first half cycle of the current pulse, the drop in electron temperature causes a rapid decrease in the plasma density. Near the end of the first half cycle of the current pulse, the intensity of the O VI $4 f-3 d$ line decreases and the line profile degrades beyond

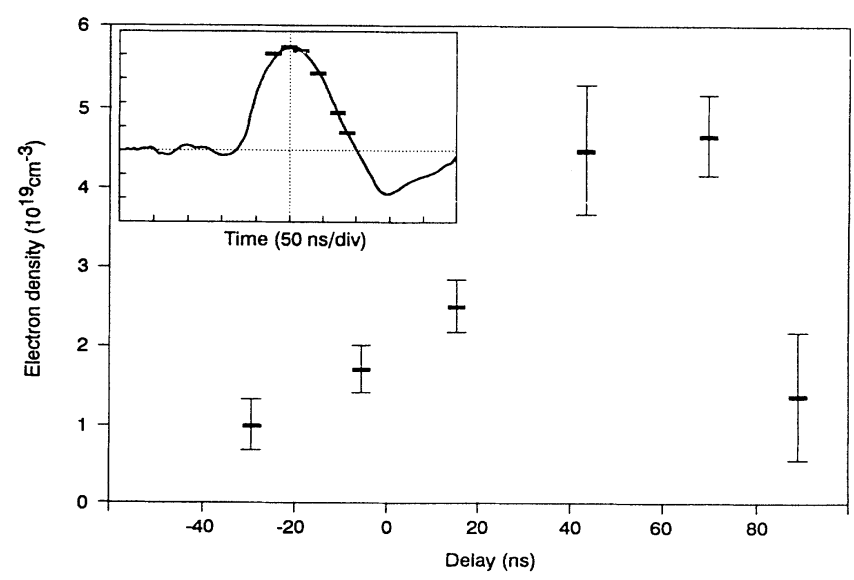

FIG. 7. Evolution of the electron density in a 1-mm-diam, 1$\mathrm{cm}$-long polyacetal capillary excited by a $15-\mathrm{kV}$ pulse. The time at which each point was measured relative to the peak of the current pulse is indicated. Each point represents the average of several measurements and the error bars correspond to one standard deviation.

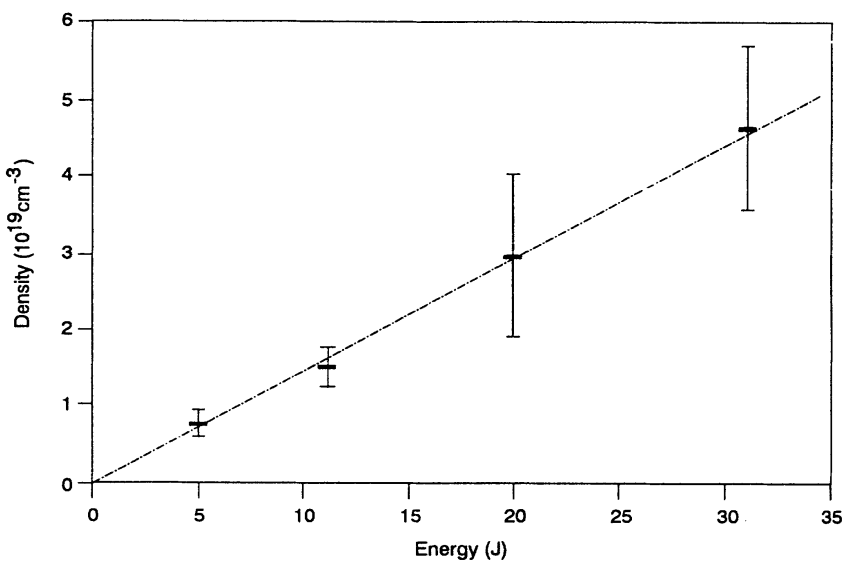

FIG. 8. Peak electron density measured in a $1.5-\mathrm{mm}$-diam, $1-\mathrm{cm}$-long capillary as a function of the capacitor bank energy.

the point at which reliable values of the density can be obtained.

As expected, a decrease in the plasma radius or an increase in the discharge energy results in a larger plasma density. The same qualitative behavior in the electrondensity evolution was also observed in discharges performed in 0.5 -mm-diam capillaries. In these smallerdiameter capillaries, however, a peak plasma density of $5 \times 10^{19} \mathrm{~cm}^{-3}$ is achieved with only $10 \mathrm{kV}$.

In capillaries $1.5 \mathrm{~mm}$ diameter, the evolution of the electron density was measured for discharge voltages of $10,15,20$, and $25 \mathrm{kV}$. In all four cases, the electron density was observed to increase during the first half cycle of the current pulse, to reach a maximum value that depends on the discharge energy at approximately $80 \mathrm{~ns}$ after the peak of the current. Figure 8 shows that the peak plasma density was measured to increase linearly with the energy of the discharge, from a value of $7.5 \times 10^{18} \mathrm{~cm}^{-3}$ for the $10-\mathrm{kV}(5 \mathrm{~J})$ discharge to $5 \times 10^{19}$ $\mathrm{cm}^{-3}$ for the $25-\mathrm{kV}(30 \mathrm{~J})$ discharge.

\section{SUMMARY AND CONCLUSIONS}

The temporal evolution of the plasma from $0.5-1.5$ mm-diam polyacetal discharges excited by 110-ns FWHM current pulses with energies between 5 and $30 \mathrm{~J}$ has been studied. The soft-x-ray spectra from these discharges are dominated by oxygen-ion lines, with the most intense emission corresponding to O VI lines. The degree of ionization of the plasma peaks shortly after the maximum of the current. Weak emission from C VI lines is observed only at the time of maximum ionization, shortly after the peak of the first half cycle of the current. Towards the end of the current pulse, the degree of ionization of the plasma decreases, the emission from $\mathrm{C} \mathrm{VI}$ vanishes, and the lines of ions with lower charge (O IV) become comparable to those of O VI. The mass accretion produced by the continuous ablation of the capillary walls maintains the plasma cold during the second half cycle of the current pulse, and during this time line emis- 
sion from highly charged ions is observed to be insignificant.

The evolution of the electron density was determined from the Stark-broadened line profile of the O VI $4 f-3 d$ line. The electron density was measured to increase during the rise of the current, to reach a maximum shortly after the time of maximum ionization, and to decrease during the last part of the first half cycle of the current pulse. The increase of the peak of the value of the plasma density with discharge energy was measured in a 1.5mm-diam discharge to vary linearly from $7.5 \times 10^{18} \mathrm{~cm}^{-3}$ for a $5-\mathrm{J}(10 \mathrm{kV})$ discharge to $5 \times 10^{19} \mathrm{~cm}^{-3}$ for a $30-\mathrm{J}(25$ $\mathrm{kV}$ ) discharge. The smaller-diameter discharges, in which a similar amount of ablated materials fills a smaller volume, produced larger plasma densities. In the 1-mmdiam capillaries, a $15-\mathrm{kV}$ discharge produced a peak density of $5 \times 10^{19} \mathrm{~cm}^{-3}$, while the same peak density was measured in the $0.5-\mathrm{mm}$-diam capillaries at a discharge voltage of $10 \mathrm{kV}$.

Emission from the CVI Balmer $\alpha$ transition was only observed near the time of maximum ionization, when the plasma density is found to be higher than the range of values in which population inversion can be obtained. Towards the end of the first half cycle of the current pulse, a time of interest for the generation of population inversions, the electron density rapidly decreases, approaching values near $1 \times 10^{19} \mathrm{~cm}^{-3}$ in the $1-\mathrm{mm}, 15-\mathrm{kV}$ discharge. However, this density decrease is accompanied by a drastic drop in the population of the most highly ionized ions. These observations suggest that a significantly faster discharge is required for the recombination of C VII ions to occur at the end of the current pulse.

\section{ACKNOWLEDGMENTS}

We want to thank Cornelius Morgan and Professor Hans Griem for frequent fruitful discussions. We are also grateful to Dr. Mario Marconi and Dr. Héctor Mancini for their collaboration. This research was supported by the U.S. Department of Energy, Advanced Energy Projects Grant No. DE-FG02-91ER12110 and the National Science Foundation Grant No. ECS 9013372.
[1] H. Conrads, Z. Phys. 444, 200 (1967).

[2] P. Bogen, H. Conrads, G. Gatti, and W. Kohlhaas, J. Opt. Soc. Am. 58 (2), 203 (1968).

[3] R. A. McCorkle, Appl. Phys. A 26, 261 (1981).

[4] J. J. Rocca, M. C. Marconi, and D. C. Beethe, Opt. Lett. 13, 565 (1988).

[5] M. C. Marconi and J. J. Rocca, Appl. Phys. Lett. 54, 2180 (1989).

[6] C. Steden and H.-J. Kunze, Phys. Lett. A 151, 534 (1990).

[7] C. A. Morgan, B. L. Welch, J. C. Moreno, and H. R. Griem, in IEEE International Conference on Plasma Science, Tampa, 1992 (IEEE, New York, 1992).

[8] R. W Lee and A. Zigler, Appl. Phys. Lett. 53, 2028 (1988).

[9] J. J. Rocca, M. C. Marconi, B. T. Szapiro, and J. Meyer, SPIE J. 1551, 275 (1991).

[10] J. J. Rocca, B. T. Szapiro, O. D. Cortázar, F. G. Tomasel, M. C. Marconi, J. Hung, and K. Floyd, in Proceedings of the 3rd International Colloquium on X-ray Lasers, Schliersee, Germany, edited by E. Fill (IOP, London, 1992).

[11] J. J. Rocca, M. C. Marconi, and F. G. Tomasel, IEEE J. Quantum Electron. 29, 182 (1993).

[12] S. Suckewer, C. H. Skinner, H. Milchberg, C. Keane, and D. Voorhees, Phys. Rev. Lett. 55, 1753 (1985).

[13] Similar measurements of the electron density based on the broadening of this transition in polyacetal discharges for voltages between 3 and $11 \mathrm{kV}$ are being conducted at the University of Maryland. See C. A. Morgan, B. L. Welch,
J. C. Moreno, and H. R. Griem, Bull. Am. Phys. Soc. 37 (6), 1459 (1992).

[14] U. Ackermann, K. H. Finken, and J. Musielok, Phys. Rev. A 31 (4), 2597 (1985).

[15] J. C. Moreno, H. R. Griem, S. Goldsmith, and J. Knauer, Phys. Rev. A 39 (11), 6033 (1989).

[16] F. Böttcher, J. Musielok, and H.-J. Kunze, Phys. Rev. A 36 (5), 2265 (1987).

[17] A. Calisti, R. Stamm, B. Talin, and R. Lee, Phys. Rev. A 42, 5433 (1990).

[18] I. P. Grant, B. J. MacKenzie, P. H. Norrington, D. F. Magers, and N. C. Pypers, Comput. Phys. Commun. 21, 207 (1980).

[19] C. A. Iglesias, J. L. Lebowitz, and D. MacGowan, Phys. Rev. A 28, 1667 (1983).

[20] R. Stamm, Y. Botzanowski, V. P. Kaftandjian, B. Talin, and E. W. Smith, Phys. Rev. Lett. 52, 2217 (1985).

[21] R. Stamm, B. Talin, E. L. Pollock, and C. A. Iglesias, Phys. Rev. A 34, 4144 (1986).

[22] S. Gleazer, N. I. Uzelac, and H.-J. Kunze, Phys. Rev. A 45, 8795 (1992) ( $3 s-3 p$ lines of $Z=6,7,8,10)$; F. Böttcher, P. Breafe, J. D. Hey, and H.-J. Kunze, Phys. Rev. A 38, 2690 (1988) (3s-3p lines of $Z=6,7,8)$; F. Böttcher, J. Musielok, and H.-J. Kunze, Phys. Rev. A 36, 2265 (1987) (3p-4d, $3 d-4 f, 4 d-5 f$, and $4 f-5 g$ lines of $Z=6,7$ )

[23] R. W. Lee, B. L. Whitten, and R. E. Strout, J. Quant. Spectrosc. Radiat. Transfer 32, 91 (1984). 